\title{
IRGM Gene
}

National Cancer Institute

\section{Source}

National Cancer Institute. IRGM Gene. NCI Thesaurus. Code C97237.

This gene is involved in inflammation. 